EPJ Web of Conferences 108, 02017 (2016)

DOI: $10.1051 /$ epjconf/201610802017

(C) Owned by the authors, published by EDP Sciences, 2016

\title{
Numerical Solution of a Nonlinear Integro-Differential Equation
}

\author{
Ján Buša ${ }^{1, a}$, Michal Hnatič ${ }^{2,3,4, b}$, Juha Honkonen ${ }^{5, c}$, and Tomáš Lučivjanský ý $^{2,6}$ \\ ${ }^{1}$ Department of Mathematics and Theoretical Informatics, FEE\&I, Technical University, Košice, Slovakia \\ ${ }^{2}$ Faculty of Sciences, P. J. Šafarik University, Košice, Slovakia \\ ${ }^{3}$ Institute of Experimental Physics SAS, Košice, Slovakia \\ ${ }^{4}$ Bogoliubov Laboratory of Theoretical Physics, JINR, 141980 Dubna, Moscow Region, Russia \\ ${ }^{5}$ Department of Military Technology, National Defence University, Helsinki, Finland \\ ${ }^{6}$ Fakultät für Physik, Universität Duisburg-Essen, D-47048 Duisburg, Germany
}

\begin{abstract}
A discretization algorithm for the numerical solution of a nonlinear integrodifferential equation modeling the temporal variation of the mean number density $a(t)$ in the single-species annihilation reaction $A+A \rightarrow \emptyset$ is discussed. The proposed solution for the two-dimensional case (where the integral entering the equation is divergent) uses regularization and then finite differences for the approximation of the differential operator together with a piecewise linear approximation of $a(t)$ under the integral. The presented numerical results point to basic features of the behavior of the number density function $a(t)$ and suggest further improvement of the proposed algorithm.
\end{abstract}

\section{Introduction}

The irreversible annihilation reaction $A+A \rightarrow \emptyset$ is a fundamental model of non-equilibrium physics. The reacting $A$ particles are assumed to perform chaotic motion due to diffusion or some external advection field such as the atmospheric eddy [1]. Many reactions of this type are observed in different chemical, biological or physical systems $[2,3]$.

In [1] the advection of a reactive scalar using a random velocity field generated either by the stochastic Navier-Stokes equation, which serves to the production of a velocity field corresponding to thermal fluctuations $[4,5]$, or by a turbulent velocity field with Kolmogorov scaling behavior [6], was studied by three of the present authors, and an integro-differential equation for the number density was derived. No influence of the reactant on the velocity field itself was assumed.

The present paper proposes a numerical solution of this integro-differential equation in the twodimensional case using regularization and then finite differences. Numerical experiments provide hints to further ways to investigate the problem.

\section{Problem Formulation}

The integro-differential equation derived in [1] (Eq. (72)) for the mean number density $a(t)$ of the chemically active molecules in anomalous kinetics of single-species annihilation reaction $A+A \rightarrow \emptyset$

\footnotetext{
a e-mail: jan.busa@tuke.sk

be-mail: hnatic@saske.sk

ce-mail: juha.honkonen@helsinki.fi
} 
writes down as

$$
\frac{\mathrm{d} a(t)}{\mathrm{d} t}=-2 \lambda u v \mu^{-2 \Delta} Z_{4} a^{2}(t)+4 \lambda^{2} u^{2} v^{2} \mu^{-4 \Delta} \int_{0}^{t} \frac{a^{2}\left(t^{\prime}\right) \mathrm{d} t^{\prime}}{\left[8 \pi u v\left(t-t^{\prime}\right)\right]^{d / 2}}, \quad a(0)=a_{0} .
$$

The integral in Eq. (1) diverges at the upper limit $t$ in space dimensions $d \geq 2$.

Starting from (1), a numerical solution is derived for the Initial Value Problem

$$
\frac{\mathrm{d} a(t)}{\mathrm{d} t}=-2 \lambda D a^{2}(t)+4 \lambda^{2} D^{2} \int_{0}^{t} \frac{a^{2}\left(t^{\prime}\right) \mathrm{d} t^{\prime}}{\left[8 \pi D\left(t-t^{\prime}\right)\right]^{d / 2}}, \quad a(0)=a_{0},
$$

which corresponds to $D=u v, \Delta=0$, and $Z_{4}=1$.

\section{Case $d=2$}

For $d=2$ the singularity at $t^{\prime}=t$ in the integral on the right hand side of Eq. (2) is divergent. This is a consequence of the UV divergences in the model above the critical dimension $d_{c}=2$. Near the critical dimension it is remedied by the UV renormalization of the model [1]. In this paper, another approach is proposed to overcome this problem. We start with the following regularization:

$$
\frac{\mathrm{d} a}{\mathrm{~d} t}=-2 \lambda D a^{2}+4 \lambda^{2} D^{2} \int_{0}^{t} \frac{a^{2}\left(t^{\prime}\right) \mathrm{d} t^{\prime}}{8 \pi\left\{D\left(t-t^{\prime}\right)+\ell^{2}\right\}}, \quad a(0)=a_{0} .
$$

Then Eq. (3) is brought to the form

$$
\frac{\mathrm{d} a}{\mathrm{~d} t}=-\alpha a^{2}+\beta \int_{0}^{t} \frac{a^{2}\left(t^{\prime}\right) \mathrm{d} t^{\prime}}{t-t^{\prime}+\gamma}
$$

where

$$
\alpha=2 \lambda D, \quad \beta=\frac{\alpha^{2}}{8 \pi D}, \quad \gamma=\frac{\ell^{2}}{D} .
$$

The resulting Eq. (4) is solved numerically using the difference method. Time discretization is done with a constant the time step $\Delta t$ :

$$
t_{k}=k \cdot \Delta t, \quad a\left(t_{k}\right)=a_{k}, \quad k=0,1,2, \ldots
$$

The discretization of the first order derivative in the left hand side uses $k$-dependent finite difference approximations:

$$
\left.\frac{\mathrm{d} a}{\mathrm{~d} t}\right|_{t=t_{k}} \approx \frac{a_{k}-a_{k-1}}{\Delta t} \quad \text { at } k=1 \quad \text { and }\left.\quad \frac{\mathrm{d} a}{\mathrm{~d} t}\right|_{t=t_{k}} \approx \frac{3 a_{k}-4 a_{k-1}+a_{k-2}}{2 \Delta t} \quad \text { at } k \geq 2 .
$$

The right-side integral approximation at $t=t_{k}$ is discretized as a sum of $k$ elementary integrals and piecewise linear approximation of the function $a(t)$ is used for the evaluation of these integrals:

$$
\begin{gathered}
\int_{0}^{t_{k}} \frac{a^{2}\left(t^{\prime}\right) \mathrm{d} t^{\prime}}{t_{k}-u+\gamma}=\sum_{i=0}^{k-1} \int_{i \cdot \Delta t}^{(i+1) \cdot \Delta t} \frac{a^{2}\left(t^{\prime}\right) \mathrm{d} t^{\prime}}{t_{k}-t^{\prime}+\gamma} \approx \\
\approx \sum_{i=0}^{k-1} \int_{i \cdot \Delta t}^{(i+1) \cdot \Delta t} \frac{\left[t^{\prime} \cdot\left(a_{i+1}-a_{i}\right) / \Delta t+(i+1) \cdot a_{i}-i \cdot a_{i+1}\right]^{2} \mathrm{~d} t^{\prime}}{t_{k}-t^{\prime}+\gamma} .
\end{gathered}
$$


The integrals in the right hand side of (7) are calculated analytically under the assumption that

$$
\begin{gathered}
\gamma=\delta \cdot \Delta t: \\
\int_{i \cdot \Delta t}^{(i+1) \cdot \Delta t} \frac{\left[t^{\prime} \cdot\left(a_{i+1}-a_{i}\right) / \Delta t+(i+1) \cdot a_{i}-i \cdot a_{i+1}\right]^{2} \mathrm{~d} t^{\prime}}{t_{k}-t^{\prime}+\gamma}= \\
=\int_{i \cdot \Delta t}^{(i+1) \cdot \Delta t} \frac{\left[\left(t^{\prime}-k \cdot \Delta t-\delta \cdot \Delta t\right) \cdot\left(a_{i+1}-a_{i}\right) / \Delta t+(k+\delta) \cdot\left(a_{i+1}-a_{i}\right)+(i+1) \cdot a_{i}-i \cdot a_{i+1}\right]^{2} \mathrm{~d} t^{\prime}}{k \cdot \Delta t-t^{\prime}+\delta \cdot \Delta t}= \\
=\int_{i \cdot \Delta t}^{(i+1) \cdot \Delta t} \frac{\left[(k+\delta-i) \cdot a_{i+1}-(k+\delta-i-1) a_{i}-\left(k \cdot \Delta t-t^{\prime}+\delta \cdot \Delta t\right) \cdot\left(a_{i+1}-a_{i}\right) / \Delta t\right]^{2} \mathrm{~d} t^{\prime}}{k \cdot \Delta t-t^{\prime}+\delta \cdot \Delta t}= \\
=\left[(k+\delta-i) a_{i+1}-(k+\delta-i-1) a_{i}\right]^{2} \cdot \int_{i \cdot \Delta t}^{(i+1) \cdot \Delta t} \frac{\mathrm{d} t^{\prime}}{k \cdot \Delta t-t^{\prime}+\delta \cdot \Delta t}- \\
-\frac{2\left[(k+\delta-i) a_{i+1}-(k+\delta-i-1) a_{i}\right] \cdot\left(a_{i+1}-a_{i}\right)}{\Delta t} \int_{i \cdot \Delta t}^{(i+1) \cdot \Delta t} \frac{\left(k \cdot \Delta t-t^{\prime}+\delta \cdot \Delta t\right) \mathrm{d} t^{\prime}}{\left(k \cdot \Delta t-t^{\prime}+\delta \cdot \Delta t\right)}+ \\
=\left[(k+\delta-i) a_{i+1}-(k+\delta-i-1) a_{i}\right]^{2} \cdot\left[-\ln \left(k \cdot \Delta t-t^{\prime}+\delta \cdot \Delta t\right)\right]_{i \cdot \Delta t}^{(i+1) \cdot \Delta t}- \\
-\frac{2\left[(k+\delta-i) a_{i+1}-(k+\delta-i-1) a_{i}\right]\left(a_{i+1}-a_{i}\right)}{\Delta t} \cdot \Delta t+\frac{\left(a_{i+1}-a_{i}\right)^{2}}{(\Delta t)^{2}} \cdot\left[\frac{\left(k \cdot \Delta t-t^{\prime}+\delta \cdot \Delta t\right)^{2}}{-2}\right]_{i \cdot \Delta t}^{(i+1) \cdot \Delta t} \\
=\left\{\left[(k+\delta-i) a_{i+1}-(k+\delta-i-1) a_{i}\right]^{2} \cdot \ln \frac{k+\delta-i}{k+\delta-i-1}-\left(k+\delta-i+\frac{1}{2}\right)\left(a_{i+1}-a_{i}\right)^{2}-2 a_{i}\left(a_{i+1}-a_{i}\right)\right\},(9) \\
i=0,1, \ldots, k-1 .
\end{gathered}
$$

Substituting the approximations (6), (7), and (9) into Eq. (4) we arrive at the quadratic equations Eq. (11) and Eq. (12) below with respect to $a_{k}$ :

$$
\begin{gathered}
0=\frac{3 a_{k}-4 a_{k-1}+a_{k-2}}{2 \Delta t}+\alpha \cdot a_{k}^{2}- \\
-\beta \cdot \sum_{i=0}^{k-2}\left\{\left[(k+\delta-i) a_{i+1}-(k+\delta-i-1) a_{i}\right]^{2} \cdot \ln \frac{k+\delta-i}{k+\delta-i-1}-\left(k+\delta-i+\frac{1}{2}\right)\left(a_{i+1}-a_{i}\right)^{2}-2 a_{i}\left(a_{i+1}-a_{i}\right)\right\}- \\
-\beta \cdot\left\{\left[(1+\delta) a_{k}-\delta a_{k-1}\right]^{2} \cdot \ln \frac{1+\delta}{\delta}-\left(1+\delta+\frac{1}{2}\right)\left(a_{k}-a_{k-1}\right)^{2}-2 a_{k-1}\left(a_{k}-a_{k-1}\right)\right\}
\end{gathered}
$$

or in the standard form

$$
\begin{gathered}
\left.\left[\alpha-\beta \cdot\left\{(1+\delta)^{2} \cdot \ln \frac{1+\delta}{\delta}-\left(\frac{3}{2}+\delta\right)\right\}\right] \cdot a_{k}^{2}+\left[\frac{3}{2 \Delta t}-\beta \cdot a_{k-1} \cdot\left\{2 \delta+1-2(1+\delta) \delta \cdot \ln \frac{1+\delta}{\delta}\right)\right\}\right] \cdot a_{k}- \\
-\frac{4 a_{k-1}-a_{k-2}}{2 \Delta t}-\beta \cdot a_{k-1}^{2} \cdot\left\{\frac{1}{2}-\delta+\delta^{2} \cdot \ln \frac{1+\delta}{\delta}\right\}- \\
-\beta \cdot \sum_{i=0}^{k-2}\left\{\left[(k+\delta-i)\left(a_{i+1}-a_{i}\right)+a_{i}\right]^{2} \cdot \ln \frac{k+\delta-i}{k+\delta-i-1}-\left(a_{i+1}-a_{i}\right)\left[(k+\delta-i)\left(a_{i+1}-a_{i}\right)+a_{i}+\frac{a_{i+1}+a_{i}}{2}\right]\right\}=0 .
\end{gathered}
$$

For the first step we get

$$
\frac{a_{1}-a_{0}}{\Delta t}+\alpha a_{1}^{2}-\beta\left\{\left[(1+\delta) a_{1}-\delta a_{0}\right]^{2} \cdot \ln \frac{1+\delta}{\delta}-\left(1+\delta+\frac{1}{2}\right)\left(a_{1}-a_{0}\right)^{2}-2 a_{0}\left(a_{1}-a_{0}\right)\right\}=0
$$



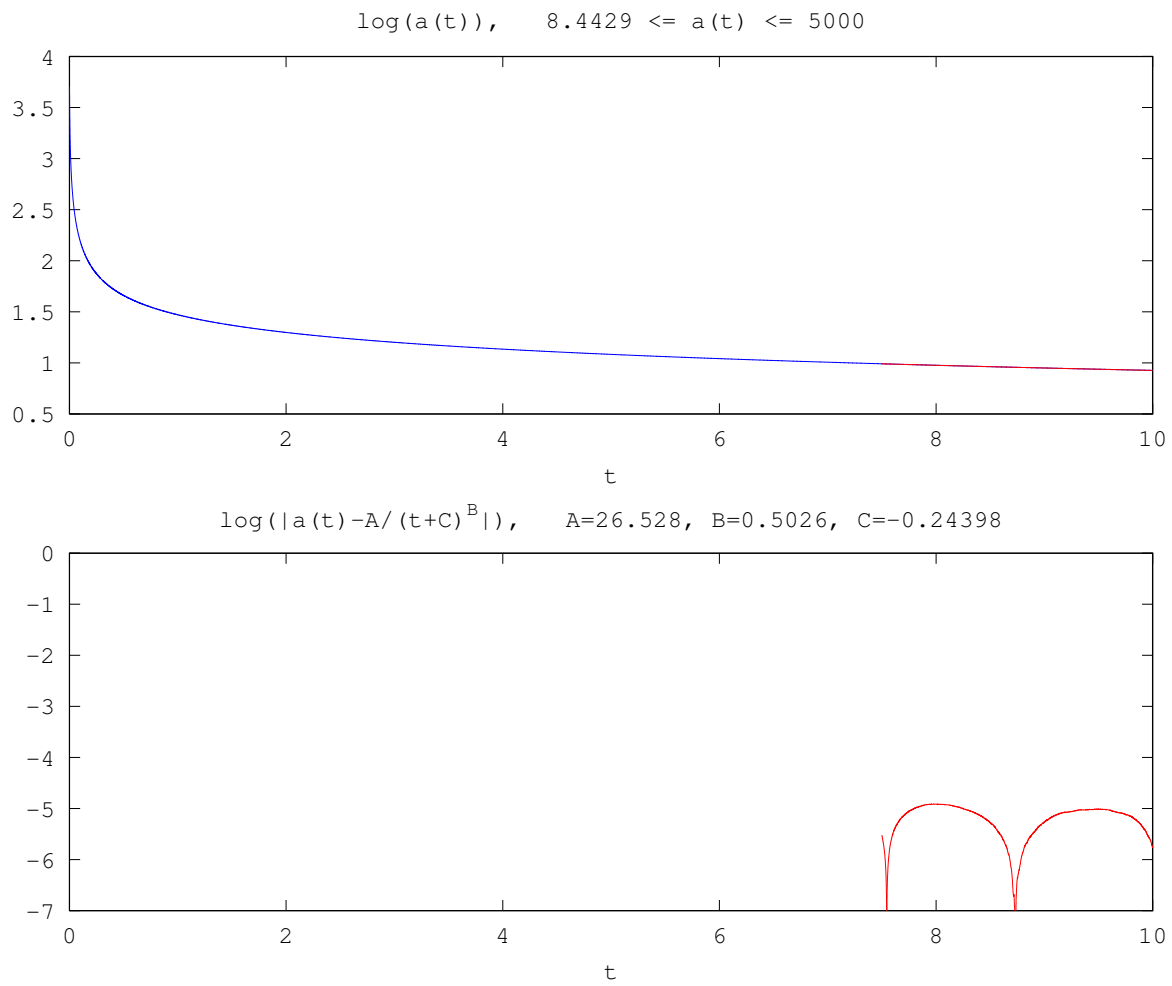

Figure 1. Logarithms of the number density $a(t)$ together with its fit (above), and the fitting error (below)

or in the standard form

$$
\begin{gathered}
\left.\left[\alpha-\beta \cdot\left\{(1+\delta)^{2} \cdot \ln \frac{1+\delta}{\delta}-\left(\frac{3}{2}+\delta\right)\right\}\right] \cdot a_{1}^{2}+\left[\frac{1}{\Delta t}-\beta \cdot a_{0} \cdot\left\{2 \delta+1-2(1+\delta) \delta \cdot \ln \frac{1+\delta}{\delta}\right)\right\}\right] \cdot a_{1}- \\
-\frac{a_{0}}{\Delta t}-\beta \cdot a_{0}^{2} \cdot\left\{\frac{1}{2}-\delta+\delta^{2} \cdot \ln \frac{1+\delta}{\delta}\right\}=0 .
\end{gathered}
$$

The algorithm starts with the known initial value $a_{0}=a(0)$ to get $a_{1}$ from the Eq. (12) and then derives successive values $a_{k}, k=1,2, \ldots, K$ by recurrence from Eq. (11).

\section{Numerical results}

Below the results for $a(0)=5000, \lambda=0.1, D=0.4$ and $\ell=0.001$ are presented. All calculations were done with a predefined uniform time step $\Delta t$.

Figure 1 up plots the variation of $a(t)$ in logarithmic scale on the interval $[0 ; 10]$ for $\Delta t=1 / 3200$ together with the logarithm of its asymptotic approximation on the interval $[7.5 ; 10]$

$$
a(t) \approx \frac{26.528}{(t-0.24389)^{0.5026}} .
$$


Table 1. Comparison of the a(t) values at selected points for different values $\Delta t$

\begin{tabular}{|l|c|c|c|r|}
\hline \multicolumn{1}{|c|}{$\Delta t$} & $t=0.01$ & $t=0.1$ & $t=1$ & \multicolumn{1}{c|}{$t=10$} \\
\hline 0.01 & 2028.8975 & 130.40166 & 41.991715 & 12.607961 \\
\hline 0.005 & 1338.5228 & 158.18202 & 35.781151 & 10.549856 \\
\hline 0.0025 & 1077.5108 & 157.67282 & 32.302138 & 9.379055 \\
\hline 0.00125 & 1062.3410 & 155.70709 & 30.581382 & 8.792275 \\
\hline 0.000625 & 1067.8149 & 154.62549 & 29.841981 & 8.538087 \\
\hline 0.0003125 & 1068.6433 & 154.18457 & 29.566128 & 8.442899 \\
\hline
\end{tabular}

In figure 1 down, the error associated to the approximation (13) on the interval $[7.5 ; 10]$ is shown. The approximation error associated to $a(t)$ is found to be smaller than $10^{-5}$.

Table 1 compares the results at selected points $t$ for the "increasing precision" obtained at decreasing values $\Delta t$ from 0.01 to 0.0003125 . Even at the finest time step $\Delta t=1 / 3200=0.0003125$ the results can not be considered to be sufficiently precise. If the step number is increasing twice, then the CPU time is increasing four times.

Assume that the difference between the numerical value $a_{\Delta t}(t)$ at the step $\Delta t$ and the "exact" value $a(t)$ is of the order $p$, i.e.,

$$
\left|a_{\Delta t}(t)-a(t)\right| \approx C \cdot(\Delta t)^{p}
$$

Writing down similar equations at $\Delta t / 2$ and $\Delta t / 4$ as well, the following empirical estimate is obtained for the "precision order" $p(t)$ :

$$
p(t) \approx \log _{2}\left|\frac{a_{\Delta t}(t)-a_{\Delta t / 2}(t)}{a_{\Delta t / 2}(t)-a_{\Delta t / 4}(t)}\right| .
$$

If this expression results in roughly constant values of $p(t)$ under the variation of both $t$ and the stepsize discretization $\Delta t$, then we may infer that the solution of (2) was obtained with the fine enough discretization stepsize $\Delta t$.

Figure 2 plots the "precision order" $p(t)$ calculated for four grid sequences: $0.01-0.005-0.0025$, $0.005-0.0025-0.00125,0.0025-0.00125-0.000625$, and $0.00125-0.000625-0.0003125$. The plot of the right points to the occurrence of the smooth $p(t)$ behavior at $t>0.5$, with orders of cca. $0.8,1.0$, 1.2 , and 1.4, respectively. The plot at the left shows that for smaller $t$-values the $p(t)$ behavior follows unstable complicated patterns.

The reported numerical results suggest that it would be reasonable to study analytically the behavior of the function $a(t)$ over small values of $t$, and to start the numerical computation from some point $t>0$. Also the use of a non-uniform time grid could be considered.

\section{Conclusions}

The numerical results presented above evidence basic features of the behavior of the number density function $a(t)$. However, further improvements of the algorithm are desirable.

It will be also interesting to try to solve the renormalized integro-differential equation also presented in [1]

$$
\begin{gathered}
\frac{\mathrm{d} a(t)}{\mathrm{d} t}=-2 \lambda u v \mu^{-2 \Delta} a^{2}(t)+2 \lambda u v \mu^{-2 \Delta} a^{2}(t)\left\{\frac{\lambda}{4 \pi}\left[\gamma+\ln \left(2 u v \mu^{2} t\right)\right]\right\}+ \\
+\frac{\lambda^{2} u v \mu^{-2 \Delta}}{2 \pi} \int_{0}^{t} \frac{\left[a^{2}\left(t^{\prime}\right)-a^{2}(t)\right] \mathrm{d} t^{\prime}}{t-t^{\prime}},
\end{gathered}
$$



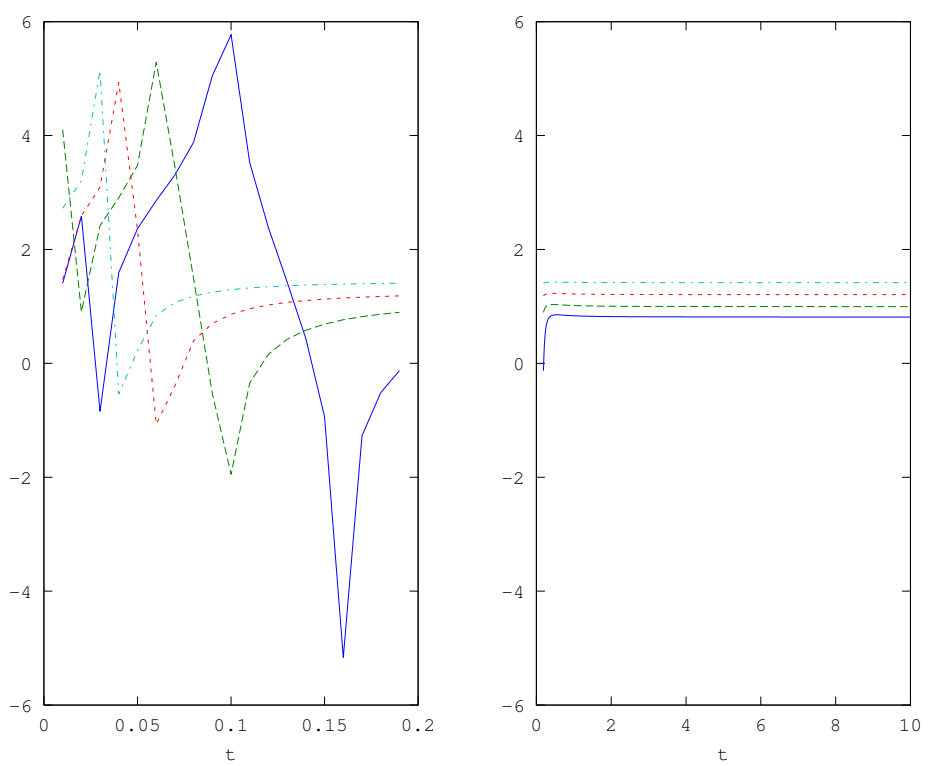

Figure 2. The $t$-dependence of the empirical precision order $p(t)$ for four cases: $0.01-0.005-0.0025, \ldots$, $0.00125-0.000625-0.0003125$

where $\Delta=(d-2) / 2$ and $\gamma \doteq 0.57721$ is the Euler constant, and to compare the results.

Another possibility is to study the behavior of the solution of the problem (1) through a sequence of solutions got at uniformly increasing $d$ values, $d \rightarrow 2^{-}$.

\section{Acknowledgements}

The work was supported by VEGA Grant 1/0222/13 of the Ministry of Education, Science, Research and Sport of the Slovak Republic.

\section{References}

[1] J. Honkonen, M. Hnatič and T. Lučivjanský, EPJ B, 86 : 214 (2013)

[2] B. Derrida, V. Hakim and V. Pasquier, Phys. Rev. Lett. 75, 751 (1995)

[3] R. Kroon, H. Fleurent and R. Sprik, Phys. Rev. E 47, 2462 (1993)

[4] D. Forster, D.R. Nelson and M.J. Stephen, Phys. Rev. Lett. 36, 867 (1976)

[5] D. Forster, D.R. Nelson and M.J. Stephen, Phys. Rev. A 16, 732 (1977)

[6] L.T. Adzhemyan, A.N. Vasil'ev and Y.M. Pis'mak, Teor. Mat. Fiz. 57, 268 (1983) 